\title{
On the coordination of motor output during visual flight control of flies
}

\author{
Johannes M. Zanker, Martin Egelhaaf, and Anne-Kathrin Warzecha \\ Max-Planck-Institut für biologische Kybernetik, Spemannstraße 38, W-7400 Tübingen, Federal Republic of Germany
}

Accepted June 17, 1991

Summary. In tethered flying houseflies (Musca domestica), the yaw torque produced by the wings is accompanied by postural changes of the abdomen and hindlegs. In free flight, these body movements would jointly lead to turning manoeuvres of the animal. By recording the yaw torque together with the lateral deflections of either the abdomen or the hindlegs, it is shown that these motor output systems act in a highly synergistic way during two types of visual orientation behavior, compensatory optomotor turning reactions and orientation turns elicited by moving objects. This high degree of coordination is particularly conspicuous for the pathway activated by moving objects. Here, orientation responses either may be induced or may fail to be generated always simultaneously in all three motor output systems. This suggests that the pathway mediating orientation turns towards objects is gated before it segregates into the respective motor control systems of the wings, the abdomen and the hindlegs.

Key words: Visual orientation - Optomotor response Motor control - Fly - Motion

\section{Introduction}

A flying animal easily may deviate from straight course in two situations. First, external disturbances such as turbulences of the air or internal asymmetries in the flight motor may force the animal to depart from its course, and the animal should try to compensate for it. Second, the animal actively may turn towards stationary or moving objects in its visual field. Both compensatory optomotor turns and turning reactions towards objects are at least partially under visual control. They are elicited by specific type of image flow on the retina. The strongest compensatory optomotor responses are induced by rotation of the complete retinal image, such as experienced by the animal during deviations from its flight course. In contrast, orientation turns towards objects may be elicited by displacements of small parts of the retinal image. This retinal flow occurs when the animal passes a nearby stationary or moving object in front of a more distant background.

How these different retinal motion patterns are transformed by the fly in either compensatory optomotor turning responses or orientation responses towards objects has been analyzed in some detail in behavioral and neurophysiological experiments. There is now good evidence that two parallel control systems are involved which differ in their sensitivity to the size of the moving stimulus (Geiger and Nässel 1982; Götz 1983a; Heisenberg and Wolf 1984; Egelhaaf 1985a-c; Bausenwein et al. 1986; Egelhaaf et al. 1988; Egelhaaf 1989; Reichardt et al. 1989; Hausen and Wehrhahn 1990). In the housefly Musca and blowfly Calliphora, the control system that mediates compensatory optomotor turning reactions ("large-field system") responds best to extended binocular stimulus patterns rotating around roughly the vertical axis of the animal. The HS-cells in the third visual ganglion have been concluded to be the corresponding output elements of the visual system (Hausen 1982a, b; for review, see Egelhaaf et al. 1988). The control system that mediates orientation turns towards objects ("small-field system") is tuned to retinal image displacements of small objects. The FD-cells that reside also in the third visual ganglion apparently represent the neuronal analogue of this control system at the output level of the visual system (Egelhaaf 1985b, c; Egelhaaf 1990). In addition to the size of the moving stimulus, the relative contribution of the large-field and small-field system to yaw torque is also influenced by the dynamic properties of stimulus motion. A comparison of the HS- and FD-cell features with the behavioral responses suggests that high frequency modulations in the output signals of the HS-cells are attenuated somewhere between the lobula plate and the final motor output. In contrast, the FD-cells mediate yaw torque also at high oscillation frequencies (Egelhaaf 1987; Egelhaaf and Borst 1990, 1991). 
How are these representations of retinal motion patterns transformed into the different types of turning reactions? In contrast to locusts (Rowell 1988), there is not much known in this respect at the cellular level, so far, in the fly. However, three motor output systems have been suggested to contribute to turns about the so-called yaw axis of flight control which is inclined about $30^{\circ}$ relative to the vertical body axis of the fly (Götz et al. 1979; Zanker 1988): (i) Differences between the mean stroke amplitudes of the two wings, (ii) abdominal deflections, and (iii) deflections of the hind legs. Do these various motor output systems act independently or synergistically in the different turning manoeuvres? To what extent are they coordinated? For example, when a pattern is moving from the left to the right in front of the fly, the average wing beat amplitude is increased on the left and decreased on the right side. In free flight, the resulting yaw torque will be supported by using frictional and gravitational force components when the abdomen and hind legs are bent simultaneously to the right side. If these output systems were not coordinated, the torque elicited by one of them would be altered by accidental signal fluctuations in the others.

Here, we examine the coordination of the 3 motor output systems contributing to compensatory turning reactions and to orientation turns towards objects in Musca. Since the large-field and small-field system which control these two types of turning responses differ mainly in their dynamical and spatial integration properties, we tuned specific stimuli in size and dynamics to address either of the two control systems.

\section{Materials and methods}

Wild type female houseflies Musca domestica (L.) from laboratory stocks were prepared as described previously (Fermi and Reichardt 1963). The head of the fly was fixed to the thorax with a mixture of wax and colophonium under light carbon dioxide anesthesia. A triangular piece of cardboard was glued to the wax just above the frontal part of the thorax. The ocelli were covered with the same mixture of wax and colophonium.

Three types of motor output were investigated. Torque: The flies were suspended from a torque compensator which prevented both rotatory and translatory movements of the animal and allowed direct measurement of the yaw torque generated by the animal (e.g. Fermi and Reichardt 1963; Götz 1964). Yaw torques measured under these conditions are mainly due to variations of the average wing beat, because in tethered flight in still air inertial or frictional forces of the body are not effective. This notion is supported by the observation that, under the stimulus conditions as used in the present study, essentially the same yaw torque responses are generated as in intact animals when the hindlegs were amputated and the abdomen was tightly fixed with wax to the thorax (Egelhaaf, unpublished observations). Abdominal deflections: The position of the abdomen tip in the equatorial plane was monitored by means of a light barrier (for details, see Zanker 1988). The light barrier was calibrated by displacing the entire fly over a known distance before and after the experiment. Leg deflections: A linescan camera (TSOpto 7120) was combined with an electronic device converting the position of a selected contour in the camera's field of view into an analogue signal. This recording technique proved to be sufficiently sensitive for monitoring the position of a single leg (in our experiments the right leg) during tethered flight.
The analogue output signals of the torque compensator, the light barrier or the linescan camera were AD-converted (DT2801) and fed into a computer (IBM-AT) and further processed as will be described in Results. For technical reasons, it was not possible to monitor simultaneously all 3 motor output variables described before. Therefore, the motor output variables were registered only in pairs, i.e. the yaw torque together with either the abdominal or the hind leg deffections.

The visual stimulation was the same as described in detail in an earlier paper (Egelhaaf 1989). In brief, the animal was positioned in the center of two concentric pattern cylinders. The outer cylinder ("ground") was opened in its rear to allow access to the abdomen and hind legs with the light barrier and linescan camera, respectively. This background pattern extended to $240^{\circ}$ in the horizontal plane of the fly's visual field. The inner stimulus pattern consisted of a cylinder segment ("figure") of $10^{\circ}$ angular width. Both, figure and ground had a vertical angular extent of $42^{\circ}$. They were covered with a vertical square-wave grating with a spatial wavelength of its fundamental of $10^{\circ}$. The fly was alternately stimulated by synchronous sinusoidal oscillations of figure and ground ("large-field motion") and by the figure oscillating alone while the background was kept stationary ("small-field motion"). While the large-field stimulus covered both eyes symmetrically, the vertical stripe mimicking small-field motion was oscillated usually in front of the right eye about a mean position of $20^{\circ}$ with respect to the frontal midline of the animal. In the experiments shown here, the oscillation amplitude was $\pm 10^{\circ}$, the oscillation frequency either $0.1 \mathrm{~Hz}$ or $1 \mathrm{~Hz}$. The experiments were carried out under open-loop conditions, i.e. the responses of the fly did not affect the visual stimulus.

\section{Results}

In a first set of experiments, it was investigated to what extent the lateral deflections of the abdomen and the yaw torque generated by the wings are coordinated during different types of visual orientation responses. Fig. 1 shows the time course of these responses during oscillatory pattern motion at frequencies of $0.1 \mathrm{~Hz}$ (left) and $1 \mathrm{~Hz}$ (right). Initially, figure and ground were moved synchronously (large-field motion) for 3 oscillation cycles. Then the ground stopped moving, while the figure continued oscillating (small-field motion) for another 3 cycles.

Let us first consider the torque response. During large-field motion, when the flies follow the pattern motion, the yaw torque can be assumed to be symmetrical around zero (Fig.1). This optomotor response would reduce the relative angular velocity between the stimulus and the eyes, if the fly was not kept stationary by the torque meter. During small-field motion the torque signal no longer oscillates arourd zero. Instead, torque is changed such that, on average, under closed-loop conditions the figure would be shifted to the front of the eyes; this means that it is positive when the figure is placed in front of the right eye. The response amplitudes differ considerably for the two oscillation frequencies. At the low oscillation frequency, the response to large-field motion is much stronger than to small-field motion. In contrast, at the high oscillation frequency, the response is more pronounced to small-field motion than to largefield motion. These results are indistinguishable from earlier experiments performed under similar stimulus conditions (Egelhaaf 1987, 1989). 
$0.1 \mathrm{~Hz}$

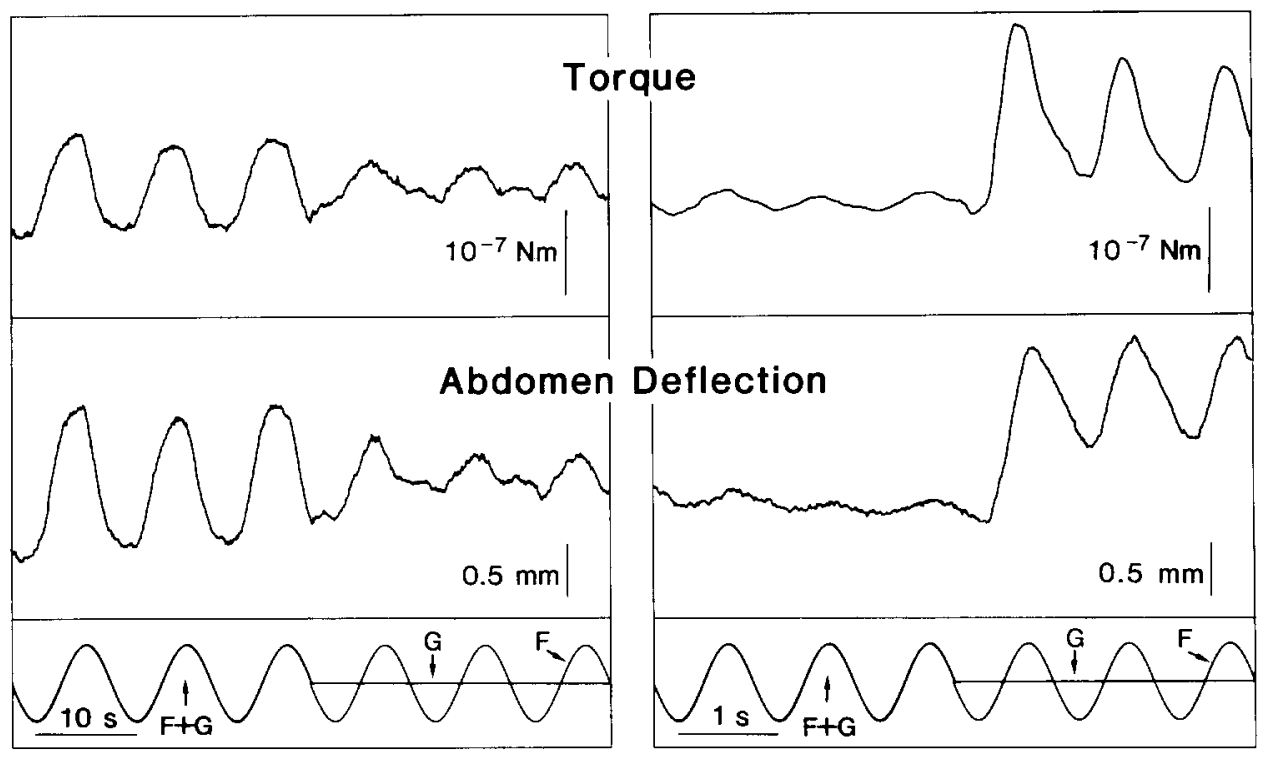

Time

Fig. 1. Simultaneously recorded yaw torque responses (upper traces) and abdomen deflections (middle traces). The flies were stimulated by oscillatory motion of a cylindrical stripe pattern (the "ground" $G$ ) and a vertical cylinder segment (the "figure" $F$ ). $F$ was placed in front of the right eye at a mean position of $20^{\circ}$ with respect to the frontal midline of the animal. The patterns were oscillated with a frequency of either $0.1 \mathrm{~Hz}$ (left diagrams) or $1 \mathrm{~Hz}$ (right diagrams). The oscillation amplitude was $10^{\circ}$. Initially $F$ and $G$ were moved synchronously for 3 cycles ("large-field motion"). Then $G$ stopped moving, while $F$ continued oscillating for another two cycles (small-field motion). Deviations of $F$ and $G$ from their respective mean positions are plotted in the bottom traces. Upward and downward slopes indicate clockwise and counter-clockwise motion, respectively. In the yaw torque response traces, upward and downward deflections indicate intended clockwise and counter-clockwise turns, respectively. Upward and downward deflections in the abdomen response traces indicate counter-clockwise and clockwise turns, respectively. The data are averages obtained from 12 flies and a total of $135(0.1 \mathrm{~Hz})$ and $318(1 \mathrm{~Hz})$ stimulus presentations. (The

The horizontal deviation of the abdomen tip from its mean position is displayed in the middle traces of Fig. 1 . During both, large-field and small-field motion, and at both oscillation frequencies, the abdomen is bent back and forth periodically with the oscillation frequency of the stimulus. For instance, the abdomen turns counterclockwise during a clockwise turn of the animal, as expected for an aerodynamic rudder. Under our stimulus conditions the overall response profiles of the yaw torque and the corresponding lateral bending of the abdomen look very similar. The amplitudes are large during smallfield motion at high oscillation frequencies as well as during large-field motion at low frequencies and comparatively small for the other conditions. However, despite this overall similarity, there are minor differences between the two motor output variables. The amplitude of the abdominal response, as compared to torque, is somewhat larger for the low oscillation frequency than for the high oscillation frequency. Furthermore, the characteristic response peaks of the lateral abdominal deflections at high oscillation frequencies are slightly
$1 \mathrm{~Hz}$

peak-to-peak amplitudes of the responses have the following S.E.M.s: Yaw torque (given in $10^{-7} \mathrm{Nm}$ ): large-field motion, $0.1 \mathrm{~Hz}: 0.15,1 \mathrm{~Hz}: 0.09$; small-field motion, $0.1 \mathrm{~Hz}: 0.07,1 \mathrm{~Hz}$ : 0.26 . Abdomen deflections (given in $\mathrm{mm}$ ): large-field motion, $0.1 \mathrm{~Hz}: 0.22,1 \mathrm{~Hz}: 0.03$; small-field motion, $0.1 \mathrm{~Hz}: 0.14,1 \mathrm{~Hz}$ : 0.12 ). Note the different time scales in the left and right diagrams. The kink in the middle of the stimulus trace does not correspond to a sudden jump of $F$, but rather is the consequence of the fact that no data were acquired while the computer triggered the motor control of the pattern to stop the ground moving. During oscillatory largefield motion both yaw torque and abdomen deflections oscillate synchronously with the pattern motion. During small-field motion and, in particular, at high oscillation frequencies the fly tries to turn towards the position of the figure. Response amplitudes are big during large-field motion at $0.1 \mathrm{~Hz}$ and small-field motion at $1 \mathrm{~Hz}$. Apart from minor differences (see text), the yaw torque and abdomen responses, under all stimulus conditions tested here, have rather similar time courses and relative amplitudes

delayed with respect to the corresponding torque responses (78 $\mathrm{ms} \pm 14.8 \mathrm{~ms} \mathrm{SEM} ; 12$ flies with 3 peaks each), and the response peaks seem to be somewhat broader. All these peculiarities of the abdominal response can be easily understood as a consequence of the big inertial mass of the abdomen. This explanation is in accordance with earlier considerations on the dynamics of abdominal deflections in Drosophila (Zanker 1988).

In a second set of experiments, the hind leg deflections were investigated under the same stimulus conditions (Fig. 2). The simultaneously recorded yaw torque traces are essentially the same as in the previous set of data. The quantitative differences in the amplitudes of the yaw torque responses displayed in Figs. 1 and 2 are well within the range of variability also found in earlier studies (Egelhaaf 1987, 1989). The middle traces of Fig. 2 show the modulations of the horizontal hind leg position. During large-field and small-field motion and at both oscillation frequencies, the hind legs are deflected periodically with the oscillation frequency of the stimulus, again in the direction opposite to the turn which would be 
$0.1 \mathrm{~Hz}$

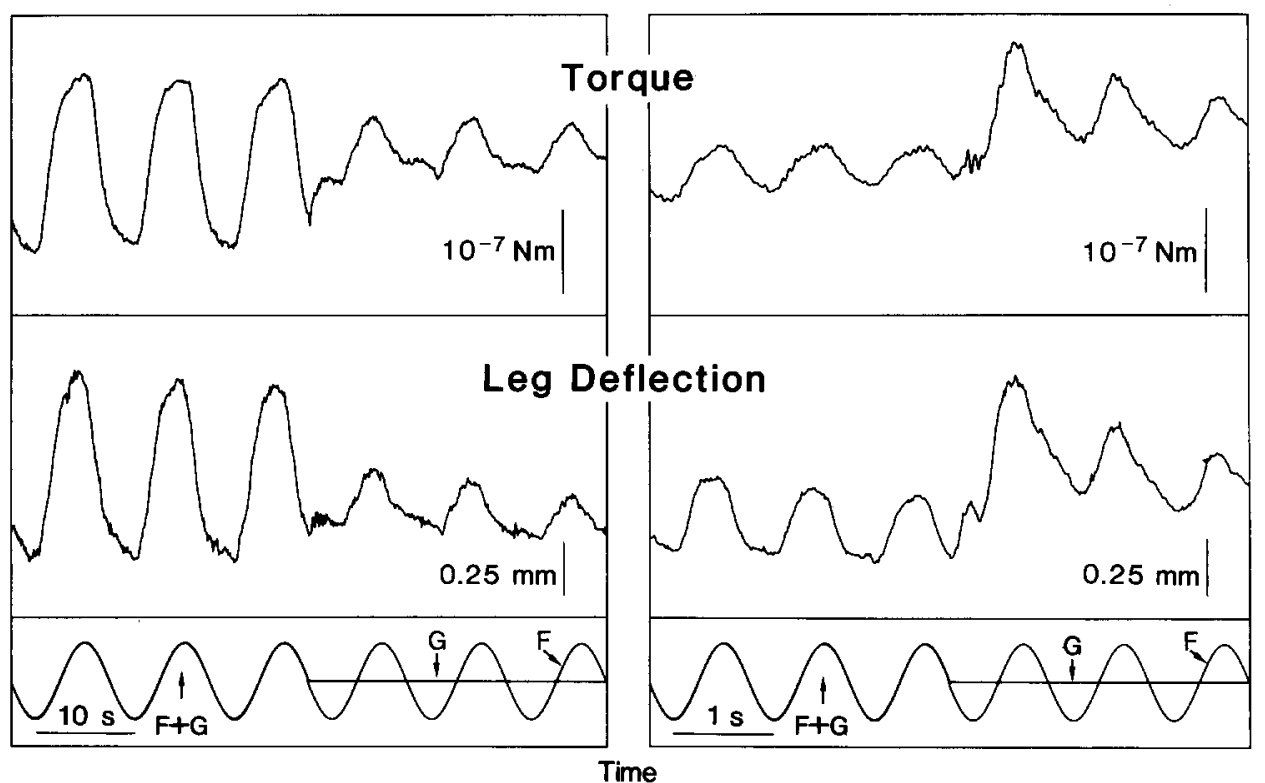

Fig. 2. Simultaneously recorded yaw torque responses (upper traces) and deflections of the right hindleg (middle traces). Stimulus conditions as described in the legend of Fig. 1. In the leg response traces, upward and downward deflections indicate movements of the legs to the right and left, respectively. The data are averages obtained from 29 flies and a total of $312(0.1 \mathrm{~Hz})$ and $407(1 \mathrm{~Hz})$ stimulus presentations. (The peak-to-peak amplitudes of the responses have the following S.E.M.s: Yaw torque (given in

generated by a freely flying fly. During large-field motion at the low oscillation frequency and during small-field motion at the high frequency the response amplitude is large, as compared to the other stimulus conditions. Despite this overall similarity with the two other motor output variables, one peculiarity of the leg deflections should be noted. At the low oscillation frequency, the average leg position during small-field motion is closer to the body of the fly (i.e. attains a smaller value, see Fig. 2) as during large-field motion. In contrast, the yaw torque response (and abdominal deflection, cf. Fig. 1) is shifted to higher values, on average. This difference may be less surprising when we assume that the average position of the leg during large-field stimulation is not necessarily the same as during straight flight, as could be concluded for the abdominal and yaw responses based on symmetry properties. If the zero level would be close to the body and, thus, deflections away from the body would be exaggerated, the average response during small-field motion could be smaller than during largefield motion. In fact, this explanation is supported by the observation that the leg position during straight flight is very close to the body, and that deflections away from the body are much more pronounced than towards it (unpublished observations).

Despite these differences in details of the response profiles of the various motor outputs, the high degree of correlation in their overall structure during visual stimulation suggests that they are controlled in the same way by visual input. So far, we have been concerned with
$10^{-7} \mathrm{Nm}$ ): large-field motion, $0.1 \mathrm{~Hz}: 0.17,1 \mathrm{~Hz}: 0.10$; small-field motion, $0.1 \mathrm{~Hz}: 0.08,1 \mathrm{~Hz}: 0.09$. Hind leg deflections (given in $\mathrm{mm}$ ): large-field motion, $0.1 \mathrm{~Hz}: 0.08,1 \mathrm{~Hz}: 0.04$; small-field motion, $0.1 \mathrm{~Hz}: 0.04 .1 \mathrm{~Hz}: 0.05$ ). The response traces, within the usual range of variability, resemble the abdomen responses shown in Fig. 1. The hind legs are deflected synchronously with the yaw torque, and the response amplitudes vary in the same way with the stimulus conditions

responses averaged over many stimulus presentations and several flies. However, the high degree of coordination of yaw torque response and postural changes of the abdomen and legs can also be observed in single recordings, although in this case the visually induced responses may be superimposed by considerable spontaneous fluctuations which need not co-vary for the different output systems. The correlation of the visually induced torque responses and the corresponding deflections of the abdomen and hind legs will be considered here only for the turning responses towards objects. Figures 3 and 4 show examples of torque responses to large-field and small-field motion at an oscillation frequency of $1 \mathrm{~Hz}$, together with the corresponding abdominal (Fig. 3) or hind leg deflections (Fig. 4). In both figures, 3 original response traces are shown which were obtained each from a single fly, during a sequence of consecutive stimulus presentations. The two flies differ with respect to the relative contributions of spontaneous signal fluctuations to the overall response and, thus, illustrate the range of variability which can be found in a typical sample of flies. Nevertheless both examples have one interesting feature in common, namely some variability in the overall response pattern which covaries in the different motor outputs. This is particularly obvious in Fig. 3 due to relatively small spontaneous signal fluctuations: Both the yaw torque and abdominal response shown in Fig. 3a resemble the averaged responses at the high oscillation frequency (Fig. 1), with small periodic modulations during large-field motion and large response peaks 

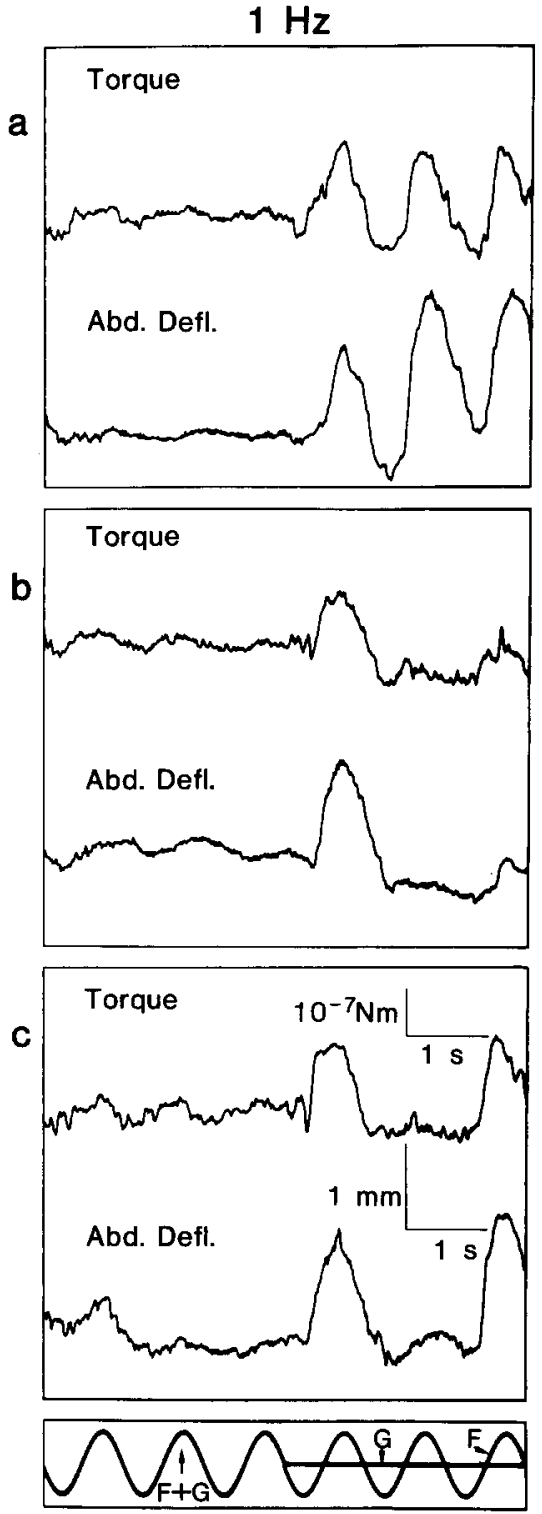

Time

Fig. 3a-c. Single traces of pairwise recorded yaw torque and abdomen responses. The fly was alternately stimulated with largefield and small-field motion. The pattern was oscillated at a frequency of $1 \mathrm{~Hz}$ and with an amplitude of $10^{\circ}$. The upper and lower diagram of each response pair represent the yaw torque and abdomen deflection, respectively. The bottom trace of the figure represents the stimulus (conventions as explained in the legend of Fig. 1). During large-field motion the fly shows only weak optomotor turning responses, in agreement with the averaged data shown in Fig. 1. During small-field motion the characteristic response peaks towards the position of the figure may be generated: (a) a peak is elicited during each stimulation cycle, (b) only the first peak is induced, (c) the second peak is missing. Hence the response peaks during small-field motion at high oscillation frequencies sometimes fail to be generated. They do this synchronously in both the yaw torque and abdomen response. As a consequence, this leads to an underestimation of the corresponding average response amplitudes

during small-field motion. However, in Fig. $3 b-c$ it is demonstrated that during small-field motion single response peaks sometimes may happen to be not generated by the fly, although the stimulus conditions are always

\section{$1 \mathrm{~Hz}$}
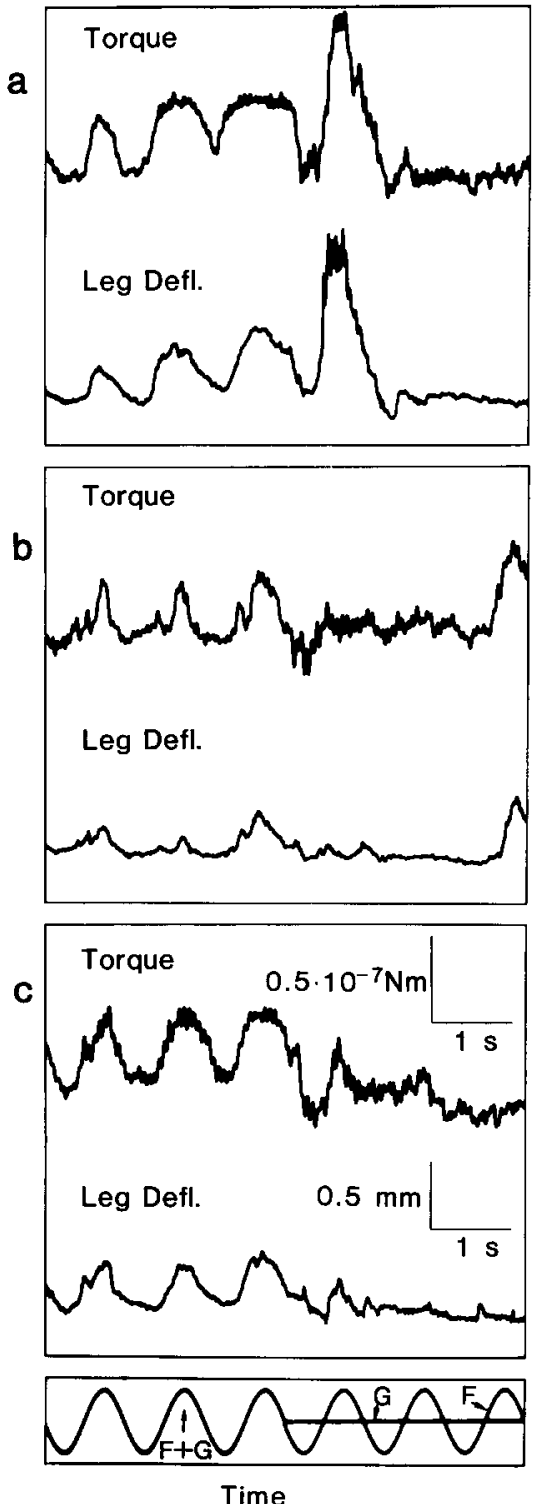

Fig. 4a-c. Single traces of pairwise recorded yaw torque and leg responses. Stimulus conditions as described in the legend of Fig. 3. As for yaw torque and abdomen deflection, both behavioral response components are highly correlated. This is despite the fact that the visually induced responses are superimposed here by noise much more than in the example shown in Fig. 3. Nevertheless, it is obvious that in (a) only the first, in (b) only the the third and in (c) no response peak is elicited during small-field motion

identical. Whereas in the example shown in Fig. 3b only the first response peak is pronounced, in the example shown in Fig. 3c only the second peak is missing. The important point is that whenever a response peak is not generated, this is the case for both the yaw torque and the abdominal response. The extraordinary degree of coordination of the different motor output systems during the given type of visual stimulation is also found for yaw torque and hind leg deflections, although in the examples displayed in Fig. 4 it is camouflaged to some extent by the much larger spontaneous signal fluctuations. 
In conclusion, the various motor output systems involved in mediating turning responses of the fly, i.e. the modulations of the average wing beat amplitude as reflected in the yaw torque, as well as the postural changes of the abdomen and hind legs, were found to be highly correlated at least as far as their visually induced components are concerned. This is not only true on average but also for the time course of single recordings.

\section{Discussion}

The virtuosic flight manoeuvres of insects require that the sensory input and motor output systems are carefully matched to each other. To control the various behavioral routines, the available sensory inputs have to be transformed appropriately and distributed to the respective sets of muscles which are involved in executing the movements. We studied the visual control of three motor output systems in the housefly Musca which appear to be involved in the control of turning responses about a roughly vertical axis of the animal. Turning responses are understood to a large extent as the consequence of flight torque generated by the two wings though the underlying aerodynamic mechanisms are obscure, still (Götz et al. 1979; Götz 1983b; Zanker 1990; Zanker and Götz 1990). In the present study the wing movements were not monitored directly. Only their immediate result was measured by means of a torque meter. In addition to modulations of the wing beat cycle, postural changes of body appendages, such as lateral abdomen and hind leg deflections, have been concluded to be involved in the turning behavior of flies (Götz et al. 1979; Zanker 1988) and locusts (Camhi 1970; Arbas 1986; Baader 1990) by shifting the center of gravity and by acting like an aerodynamic rudder.

In the present study, the coordination of yaw torque, abdominal and hind leg deflections was analyzed for two types of visually induced flight manoeuvres in tethered flight, compensatory turning responses and turning responses towards objects. It was shown that the yaw torque generated by the wings, the lateral bending of the abdomen and the hind leg deflections are elicited in a highly correlated manner during both types of turning reactions. This is not only true for averaged responses but also for the time courses of individual response traces. Hence, the different motor output systems discussed here do not appear to be specialized for mediating particular functional types of turns. It should be noted that this is by no means self-evident. For instance, the different steering muscles of the wings which are assumed to be involved in mediating yaw torque (for review, see Heide 1983) are functionally specialized: Whereas some direct flight muscles were concluded to mediate mainly orientation turns towards objects, another type of steering muscle is also responsible for compensatory optomotor responses (Egelhaaf 1989). Hence, there is not only diversification between the muscular systems of the wings and the body appendages, but also within the wing beat steering muscles. On the other hand, the time course of the various motor output systems, such as postural changes of abdomen and hind legs and yaw torque, differ only in minor details from each other when the fly performs various types of turning manoeuvres. What seems to be rather simple at the level of the final motor output, thus may be a complex problem for the underlying neuronal system which has to recruit the appropriate sets of muscles in a well organized and orderly fashion.

As mentioned in the Introduction, compensatory turning responses and orientation turns towards objects appear to be mediated by two parallel control systems, the "large-field" and the "small-field system" which can be attributed to two functional classes of output cells of the optic lobes, the HS- and the FD-cells. Before the specific information extracted by the HS- and FD-cells is distributed to the various motor output systems, it is further processed in different ways. (i) A kind of temporal low-pass filter was proposed (Egelhaaf 1987; Egelhaaf and Borst 1990, 1991) for the pathway of the largefield system which attenuates the high frequency components in the HS-cell signals. Although nothing is known so far about the neuronal nature of the temporal low-pass filter, it should be located at some stage before the motor commands segregate to the wings, the abdomen, and the hind legs, because all 3 have the same dynamical properties. (ii) The fly does not always respond to small-field motion with the motor output systems investigated here. Often single response peaks are omitted. Although this may be sometimes camouflaged by signal components which appear to be independent of visual stimulation, the response peaks seem to fail simultaneously in all the motor output systems considered here. From this observation two conclusions can be drawn. First, the responses are gated before the information on small-field motion segregates to the motor control centers of the wings, the abdomen and the hind legs. Second, the signals carried by the small-field system seem to be gated by some other determinant than visual information. Wind input may play a modulatory role since, at least in some flies, the responses to visual small-field motion occur more reliably during simultaneous wind stimulation of the tethered flying animal (Egelhaaf, unpublished observations). However, other factors which are not related to sensory input and are hard to characterize experimentally, such as the internal state of the animal, are likely to play an important role in gating the signals carried by the small-field system. In order to understand the neuronal basis of this processing, the descending neurones conveying information from the optic lobes to the thoracic motor centers are of particular interest. In contrast to locusts (for review, see Rowell 1988), however, not much is known about their physiology in flies, despite an extensive anatomical description (e.g. Strausfeld 1989; Milde and Strausfeld 1990; Strausfeld and Gronenberg 1990; Gronenberg and Strausfeld 1990).

The gating in the pathway for small-field motion must not be confounded with two other gating phenomena which have been described previously. First, the gating of any visual input of the wing steering muscles by signals of the flight motor. For instance, the wing steering muscles of flies (Heide 1975), the abdominal reactions in 
locusts (Camhi 1970), or the activity of flight motoneurons in locusts (Reichert et al. 1985) are only responsive to sensory input as long as the animal is flying. As a consequence of this gating process, the various motor output systems involved in course control are active only during flight. Second, the visual input has been found to gate wingbeat synchronous proprioceptive afferences which phasically influence the motor control systems of the wings, both in flies (Heide 1971, 1974, 1975, 1983) and in locusts (Reichert et al. 1985; Reichert and Rowell 1985). The gating of the small-field system as described in the present study differs from these two other gating phenomena in that the gating signal can be neither attributed to the activity of the flight motor per se nor directly to sensory afferences.

In contrast to $\mathrm{Musca}$, the lateral abdominal deflections in Drosophila in response to both large-field and small-field motion have the same dynamical properties (Zanker 1988; Zanker and Quenzer 1988). In Drosophila both behavioral response components are most sensitive to low oscillation frequencies, as has been found in Musca for the yaw torque, abdominal and hind leg responses to large-field but not to small-field motion (Egelhaaf 1987, and present study). There are several possible explanations for this discrepancy. (i) There is no specific small-field system in Drosophila. (ii) The information about small-field motion is not transmitted to the abdominal motor system in Drosophila. (iii) The smallfield system could not be activated in Drosophila under the stimulus conditions used in these studies. (iv) The small-field system of Drosophila has different temporal transfer properties than that of Musca. If one of the first three hypotheses were correct, the abdominal responses of Drosophila obtained during small-field motion are mediated by the large-field system alone. This could well be the case, since, at least in Calliphora, the HS-cells respond not only to large-field motion but also, though with smaller amplitudes, to small-field motion (Hausen 1982a, b). However, the first of the above hypotheses can probably be discarded immediately. There is ample evidence that in Drosophila there is at least another control system, in addition to the one mediating compensatory optomotor turning responses, which responds best to relatively small moving targets (e.g. Götz 1983a; Heisenberg and Wolf 1984; Bausenwein et al. 1986). However, in these studies this control system has been approached from a rather different perspective than in the attempts to characterize the small-field system in the larger flies. This makes it hard to compare the visual input organization and, in particular, the dynamical response properties of the small-field system in Musca and Calliphora with its hypothetical counterpart in Drosophila.

In conclusion, our behavioral data reveal that, at least in Musca, the various motor output systems which are involved in generating turning responses of the animal about its yaw axis are coupled to a high degree. This was shown here for two types of visually induced turning manoeuvres, namely for compensatory optomotor responses and for orientation turns towards objects. It has to be analyzed whether the motor output systems are coordinated to the same extent during other manoeuvres, for example during spontaneous turns or turning responses induced by other sensory modalities.

Acknowledgements. We thank Silke Marcinowski for excellent technical assistance. We also thank Alexander Borst and Werner Reichardt for helpful discussions during the course of the experiments and for critically reading the manuscript. The figures are due to the skill of Anke Wildemann.

\section{References}

Arbas EA (1986) Control of hindlimb posture by wind-sensitive hairs and antennae during locust flight. J Comp Physiol A $159: 849-857$

Baader A (1990) The posture of the abdomen during locust flight: Regulation by steering and ventilatory interneurones. J Exp Biol 151:109-131

Bausenwein B, Wolf R, Heisenberg M (1986) Genetic dissection of optomotor behavior in Drosophila melanogaster. Studies on wild-type and the mutant optomotor-blind H31. J Neurogenetics 3:87-109

Camhi JM (1970) Yaw-correcting postural changes in locusts. J Exp Biol 52:519-531

Egelhaaf M (1985a) On the neuronal basis of figure-ground discrimination by relative motion in the visual system of the fly. I. Behavioural constraints imposed on the neuronal network and the role of the optomotor system. Biol Cybern 52:123-140

Egelhaaf M (1985b) On the neuronal basis of figure-ground discrimination by relative motion in the visual system of the fly. II. Figure-Detection Cells, a new class of visual interneurones. Biol Cybern 52:195-209

Egelhaaf M (1985c) On the neuronal basis of figure-ground discrimination by relative motion in the visual system of the fly. III. Possible input circuitries and behavioural significance of the FD-Cells. Biol Cybern 52:267-280

Egelhaaf M (1987) Dynamic properties of two control systems underlying visually guided turning in house-flies. J Comp Physiol A 161:777-783

Egelhaaf M (1989) Visual afferences to flight steering muscles controlling optomotor response of the fly. J Comp Physiol A $165: 719-730$

Egelhaaf M (1990) Spatial interactions in the fly visual system leading to selectivity for small-field motion. Naturwissenschaften $77: 182-185$

Egelhaaf M, Borst A (1990a) Bewegungswahrnehmung und visuelle Orientierung bei Fliegen. Naturwissenschaften 77:366-377

Egelhaaf M, Borst A (1991) Motion computation and visual orientation in flies. In: Barnes WJP (ed) Sensory guidance in arthropod behaviour. Manchester University Press, Manchester, in press

Egelhaaf M, Hausen K, Reichardt W, Wehrhahn C (1988) Visual course control in flies relies on neuronal computation of object and background motion. Trends Neurosci 11:351-358

Fermi G, Reichardt W (1963) Optomotorische Reaktionen der Fliege Musca domestica. Abhängigkeit der Reaktion von der Wellenlänge, der Geschwindigkeit, dem Kontrast und der mittleren Leuchtdichte bewegter periodischer Muster. Kybernetik 2: 15-28

Geiger G, Nässel DR (1982) Visual processing of moving single objects and wide-field patterns in flies: Behavioural analysis after laser-surgical removal of interneurons. Biol Cybern 44: $141-149$

Geiger G, Poggio T (1977) On head and body movements of flying flies. Biol Cybern 25:177-180

Gronenberg W, Strausfeld NJ (1990) Descending neurons supplying the neck and flight motor of Diptera: Physiological and anatomical characteristics. J Comp Neurol 302:973-991

Götz KG (1964) Optomotorische Untersuchung des visuellen Systems einiger Augenmutanten der Fruchtfliege Drosophila. Kybernetik 2:77-92 
Götz KG (1983a) Genetischer Abbau der visuellen Orientierung bei Drosophila. Verh Dtsch Zool Ges 76:83-99

Götz KG (1983b) Bewegungssehen und Flugsteuerung bei der Fliege Drosophila. In: Nachtigall W (ed) BIONA report. Akademie Wissenschaften Literatur Mainz, G Fischer, Mainz Stuttgart New York, pp 21-34

Götz KG, Hengstenberg B, Biesinger R (1979) Optomotor control of wing beat and body posture in Drosophila. Biol Cybern $35: 101-112$

Hausen K (1982a) Motion sensitive interneurons in the optomotor system of the fly. I. The Horizontal Cells: Structure and signals. Biol Cybern 45: 143-156

Hausen K (1982b) Motion sensitive interneurons in the optomotor system of the fly. II. The Horizontal Cells: Receptive field organization and response characteristics. Biol Cybern 46: 67-79

Hausen K, Wehrhahn C (1990) Neural circuits mediating visual flight in flies. II. Separation of two control systems by microsurgical brain lesions. J Neurosci 10:351-360

Heide G (1971) Die Funktion der nicht-fibrillären Flugmuskeln von Calliphora. Teil II: Muskuläre Mechanismen der Flugsteuerung und ihre nervöse Kontrolle. Zool Jb Physiol 76:99-137

Heide G (1974) The influence of wingbeat synchronous feedback on the motor output systems in flies. Z Naturforsch 29c:739744

Heide G (1975) Properties of a motor output system involved in the optomotor response in flies. Biol Cybern 20:99-112

Heide G (1983) Neural mechanisms of flight control in Diptera. In: Nachtigall W (ed) BIONA report. Akad Wissenschaften Literatur Mainz. G Fischer, Mainz Stuttgart New York, pp 3552

Heisenberg M, Wolf R (1984) Vision in Drosophila. Springer, Berlin Heidelberg New York, Tokyo
Milde JJ, Strausfeld NJ (1990) Cluster organization and response characterictics of the giant fiber pathway of the blowfly $\mathrm{Cal}$ liphora erythrocephala. J Comp Neurol 294:59-75

Reichardt W, Egelhaaf M, Guo A (1989) Processing of figure and background motion in the visual system of the fly. Biol Cybern $61: 327-345$

Reichert H, Rowell CHF (1985) Integration of nonphaselocked exteroceptive information in the control of rhythmic flight in the locust. J Neurophysiol 53:1201-1218

Reichert H, Rowell CHF, Griss C (1985) Course correction circuitry translates feature detection into behavioural action in locusts. Nature 315: 142-144

Rowell CHF (1988) Mechanisms of flight steering in locusts. Experientia $44: 389-395$

Strausfeld NJ (1989) Beneath the compound eye: Neuroanatomical analysis and physiological correlates in the study of insect vision. In: Stavenga DG, Hardie RC (eds) Facets of vision. Springer, Berlin Heidelberg New York, pp 317-359

Strausfeld NJ, Gronenberg W (1990) Descending neurons supplying the neck and flight motor of Diptera: Organization and neuroanatomical relationships with visual pathways. J Comp Neurol 302:954-972

Zanker JM (1988) How does lateral abdomen defiection contribute to flight control of Drosophila melanogaster? J Comp Physiol A $162: 581-588$

Zanker JM (1990) The wing beat of Drosophila melanogaster III. Control. Phil Trans R Soc Lond B 327:45-64

Zanker JM, Götz KG (1990) The wing beat of Drosophila melanogaster II. Dynamics. Phil Trans R Soc Lond B 327: 19.44

Zanker JM, Quenzer T (1988) Abdominal deflections elicited by stripe movement. In: Elsner N, Barth FG (eds) Sense organs: Interfaces between environment and behaviour. $\mathrm{G}$ Thieme, Stuttgart New York, pp 132 